\title{
Hierarchical Layouts of Directed Graphs in Three Dimensions
}

\author{
Seok-Hee Hong ${ }^{1,2}$ and Nikola S. Nikolov ${ }^{1,3}$ \\ 1 IMAGEN Program, National ICT Australia Ltd. \\ 2 School of IT, University of Sydney, NSW, Australia \\ 3 Department of CSIS, University of Limerick, Limerick, Republic of Ireland \\ \{seokhee.hong, nikola.nikolov\}@nicta.com.au
}

\begin{abstract}
We introduce a new graph drawing convention for 3D hierarchical drawings of directed graphs. The vertex set is partitioned into layers of vertices drawn in parallel planes. The vertex set is further partitioned into $k \geq 2$ subsets, called walls. The layout consists of a set of parallel walls which are perpendicular to the set of parallel planes of the layers. We also outline a method for computing such layouts and introduce four alternative algorithms for partitioning the vertex set into walls which address different aesthetic requirements 1
\end{abstract}

\section{Introduction}

The visual representation of hierarchically organised data has application in areas such as Social Network Analysis, Bioinformatics, Software Engineering, etc. Hierarchies are commonly modeled by directed graphs (digraphs) and thus visualised by algorithms for drawing digraphs. Most of the research effort in this area has been related to improvements of various aspects of the Sugiyama method, the most popular method for creating 2D layered drawings of digraphs [4, 9].

The increasing availability of powerful graphic displays opens new opportunities for developing new methods for 3D graph drawing. There is evidence that 3D graph layouts combined with novel interaction and navigation methods make graphs easier to comprehend by humans and increase the efficiency of task performance on digraphs [10.

However, there has been relatively little research on drawing digraphs in 3D. One of the known approaches is the method of Ostry which consists of computing a layered drawing in 2D and then wrapping it around a cone or a cylinder 8 . Another approach is the method used in the graph drawing system GIOTTO3D [5] which is conceptually different from the Sugiyama method. GIOTTO3D employs a simple 3-phase algorithm for producing 3D layered drawings of digraphs. In the first phase a planarisation method is used to draw the graph in $2 \mathrm{D}$; in the second phase vertices and edges are assigned z-coordinates so that all edges point

\footnotetext{
${ }^{1}$ An online gallery with examples of our 3D hierarchical layouts is available at http://www.cs.usyd.edu.au/ visual/valacon/gallery/3DHL.
} 


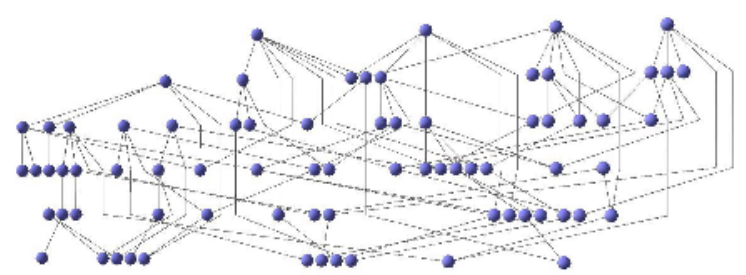

(a) $2 \mathrm{D}$

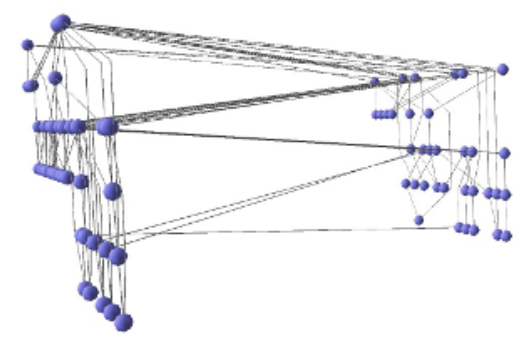

(b) Zig-zag

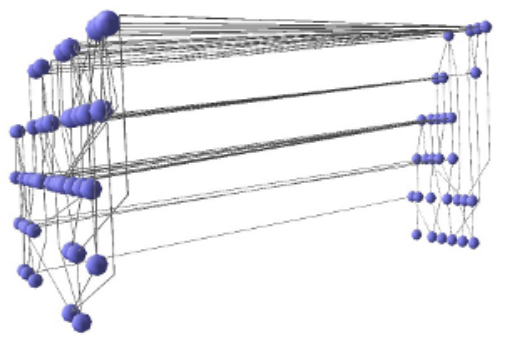

(c) Dominating wall

Fig. 1. A 2D layered drawing (a) compared to 3D layered layouts of the same graph with two parallel walls (b) and (c)

into the same direction and the total edge span is minimised; and at the third phase the shape of the vertices and the edges is determined.

This paper presents a method for layered drawing of digraphs in 3D which extends the Sugiyama method. We generalise and extend the work presented in our previous paper [6. In summary, we propose an extra step after the layer assignment of the 2D Sugiyama method. It consists of partitioning the vertex set into subsets, called walls. Any subset of the vertex set can be a wall. We propose that layers occupy parallel planes with all edges pointing in the same direction. Walls also occupy parallel planes which are perpendicular to the layer planes. Each pair of a wall and a layer intersect into a set of vertices placed along the line which is the intersection of the corresponding wall and layer planes. As a result each wall contains a 2D layered drawing. Examples of such a layout can be seen in Figures 1 and 2 .

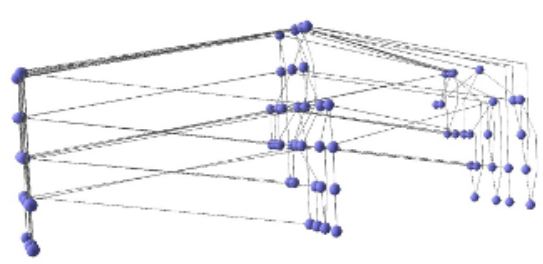

(a) According to C4

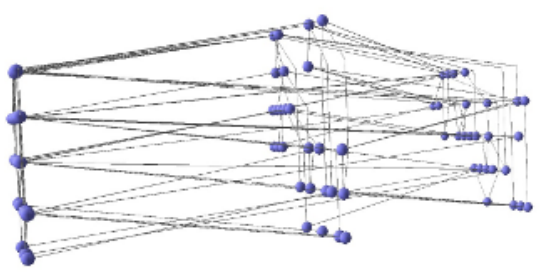

(b) According to both criteria C1 and C4

Fig. 2. Examples of 3D hierarchical layouts with $k>2$ parallel walls 
The motivation behind the proposed drawing convention consists of the following points:

- A 3D layered drawing of a digraph allows the employment of specific 3D navigation and interaction techniques and decreases visual complexity. For example, each wall can be viewed separately, the camera may move along edges between the walls, etc.

- Partitioning the hierarchy into a set of walls each containing a smaller 2D hierarchy allows us to draw the smaller 2D hierarchies efficiently with fast heuristics or even exact algorithms which generally would perform worse if employed for drawing the whole graph as a 2D hierarchy.

- Drawing the 3D hierarchy as a set of 2D hierarchies utilises the extensively developed techniques for drawing hierarchies in $2 \mathrm{D}$.

Our method can be applied to any digraph, such as a class hierarchy that originates from a Software Engineering application, or a hierarchical relationship in a social network, etc. In particular, we report experiments with some of the graphs in the Rome data set [3].

The paper is organised as follows: in the next section we introduce some definitions and an outline of the Sugiyama method for drawing digraphs in 2D. Then in Section 3 we describe our 3D extension to the Sugiyama method. In Section 4 we show some drawings of digraphs computed with our method and we compare them to the corresponding 2D drawings. Finally, in Section 5] we draw some conclusions from this work.

\section{Terminology}

The Sugiyama method for layered digraph drawing consists of four steps. The first step is to remove all directed cycles from the graph by inverting the direction of some edges. In the second step the vertices of the digraph are partitioned into layers. Let $G=(V, E)$ be a digraph without directed cycles. We denote the set of all immediate predecessors of vertex $\mathrm{v}$ by $N^{-}(v)=\{u:(u, v) \in E\}$, and the set of all its immediate successors by $N^{+}(v)=\{u:(v, u) \in E\}$. A layering of $G$ is defined as an ordered partition $L=\left\{L_{1}, L_{2}, \ldots, L_{h}\right\}$ of its vertex set into $h$ subsets, called layers, such that $(u, v) \in E$ with $u \in L_{i}$ and $v \in L_{j}$ implies $j<i$. A digraph with a layering is a layered digraph. A layering is proper if all edges are between vertices in adjacent layers. If this is not the case then after the second step of the Sugiyama method dummy vertices which subdivide long edges, i.e. edges which connect vertices in non-adjacent layers, are introduced. Formally, for each edge $e=(u, v)$ with $u \in L_{i}, v \in L_{j}$, and $j<i-1$, we introduce $i-j-1$ dummy vertices $d_{j+1}^{e}, d_{j+2}^{e}, \ldots, d_{i-1}^{e}$ into layers $L_{j+1}, L_{j+2}, \ldots, L_{i-1}$, respectively. We also replace edge $e$ by edges $\left(u, d_{i-1}^{e}\right),\left(d_{i-1}^{e}, d_{i-2}^{e}\right), \ldots,\left(d_{j+2}^{e}, d_{j+1}^{e}\right),\left(d_{j+1}^{e}, v\right)$.

In the third step a linear order is established for the vertices in each layer. And in the last fourth step $x$ - and $y$-coordinates of all vertices are decided as well as the shape of the edges. Various algorithms, which emphasise different properties of the drawing, have been suggested for each step of the Sugiyama method [2]. 


\section{3D Layered Drawing of Directed Graphs}

In this section we propose a $3 \mathrm{D}$ extension to the Sugiyama method. In summary, we introduce a new step called wall assignment which further partitions the layer into a set of $k>1$ subsets, called walls, after the layering step. Our method is outlined in Algorithm 1 ,

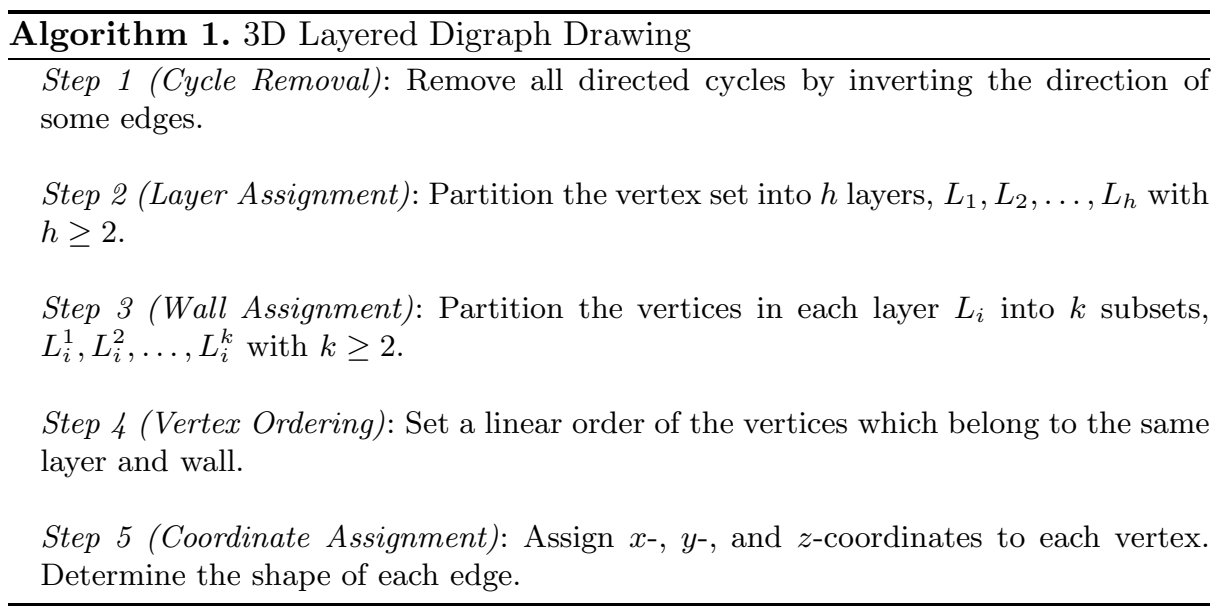

Layers occupy parallel planes and each layer $L_{i}$ is partitioned into $k$ subsets, $L_{i}^{1}, L_{i}^{2}, \ldots, L_{i}^{k}$. The vertices placed in the $j^{t h}$ group of each layer form a wall. That is, the set $W^{j}=\left\{L_{1}^{j}, L_{2}^{j}, \ldots, L_{h}^{j}\right\}$ is the $j^{\text {th }}$ wall. There are $k$ walls in total, each wall occupies a plane, and the walls occupy $k$ parallel planes which are perpendicular to the $h$ planes of the layers. In addition, we require all dummy vertices along the same long edge to be in the same wall in order to avoid more than one bend outside the walls which contain the endpoints of the edge.

Since we perform the wall-assignment step after the introduction of dummy vertices we assume that $G=(V, E)$ is a proper layered digraph with a layering $L=\left\{L_{1}, L_{2}, \ldots, L_{h}\right\}$, i.e. each edge connects vertices in adjacent layers. By partitioning the vertices into $k \geq 2$ walls we partition the edge set of a digraph into two subsets: intra wall edges and inter wall edges. Intra wall edges are edges with both endpoints in the same wall, and inter wall edges are edges with endpoints in different walls. The span of an inter wall edge is the absolute value of the difference between the numbers of the two walls which contain the endpoints of that edge. Note that each inter wall edge has at least one endpoint which is not a dummy vertex because we require all dummy vertices along the same long edge to be in the same wall.

The partition of the original vertex set into $k$ walls may originate from the digraph's application domain. They might be the clusters of a given clustered graph. If no such partition is given then the vertex set can be partitioned into $k$ walls according to the following optimisation criteria: 
- C1. Even distribution of vertices among walls, i.e. balanced partition of the vertex set into walls.

- C2. As few as possible inter wall edges for avoiding occlusion in the 3D space.

- C3. As few as possible crossings between inter wall edges in the projection of the drawing into a plane which is orthogonal to both the layer planes and the wall planes.

- C4. The sum of the spans of inter wall edges should be minimised.

- C5. As few as possible crossings between intra wall edges.

These criteria are designed to express the properties of layouts with low visual complexity. They give rise to some hard optimisation problems which require the development of efficient algorithms. In our previous work we have proposed an algorithm for partitioning the vertex set into two walls according to $\mathrm{C} 1$ and $\mathrm{C} 2$. In the remainder of this section we propose two new methods of partitioning the vertex set into two walls according to C3, and two versions of a method for partitioning the vertex set into $k$-walls according to $\mathrm{C} 4$ and $\mathrm{C} 1$.

\subsection{Two-Wall Partitions}

The following two algorithms for partitioning the vertex set into two walls are designed to have all inter wall edges arranged in a particular pattern such that C3 is satisfied. We call them zig-zag wall partition and dominating wall partition respectively. Both algorithms scan all layers one by one from bottom to top and partition each of them into two subsets. We start with a random balanced partition of the first layer that contains more than one vertex. Each next layer $L_{i}$ is partitioned into $L_{i}^{1}$ and $L_{i}^{2}$ such that $L_{i}^{1} \cup L_{i}^{2}=L_{i}$ and $L_{i}^{1} \cap L_{i}^{2}=\phi$ based on the partition of layer $L_{i-1}$.

The zig-zag wall partition and the dominating wall partition differ in the way layer $L_{i}$ is divided into two subsets based on the already given partition of layer $L_{i-1}$ for $i \geq 2$. The zig-zag wall partition scans all the vertices in layer $L_{i}$ and partitions it into $L_{i}^{1}$ and $L_{i}^{2}$ by applying different strategies for even and odd layers. If $i$ is an even number then $u \in L_{i}$ will be always assigned to $L_{i}^{2}$ except in the case when $u$ has no neighbours in $L_{i-1}^{2}$. In that case $u$ will be assigned to $L_{i}^{1}$. That is, a vertex is assigned to $L_{i}^{1}$ if and only if it has all its immediate successors in $L_{i-1}^{1}$. Note that this is done after the insertion of dummy vertices and all the immediate successors of $u$ are in layer $L_{i-1}$. If $i$ is an odd number then $u \in L_{i}$ is assigned to $L_{i}^{2}$ if and only if all its immediate successors are assigned to $L_{i-1}^{2}$. As a result the inter wall edges form a zig-zag pattern between the two walls. The zig-zag wall partition is presented as Algorithm 2, An example layout is shown in Figure 1(b).

The idea of the dominating wall partition is all the inter wall edges have their origin in the same wall, which we call the dominating wall. We assume that wall $W^{1}$ is the dominating wall, i.e. the wall that consists of $L_{1}^{1}, L_{2}^{1}, \ldots, L_{h}^{1}$. Vertex $u \in L_{i}$ is assigned to $L_{i}^{2}$ if and only if it has no neighbours in $L_{i-1}^{1}$. The dominating wall partition of layer $L_{i}$ is presented as Algorithm 3 . An example layout is shown in Figure 1(c). 

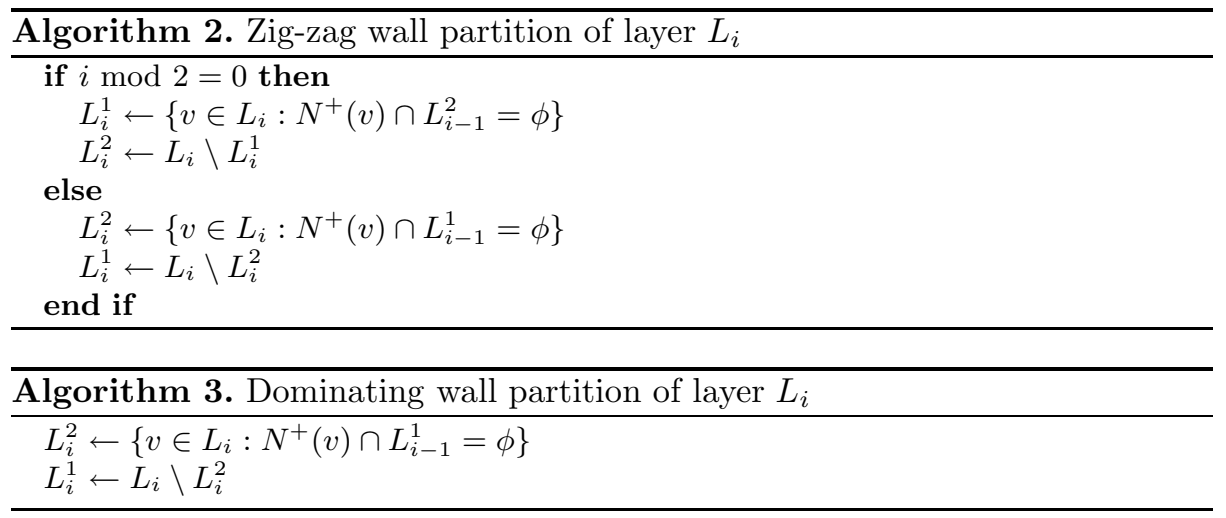

It is easy to see that both the zig-zag wall partition and the dominating wall partition place all dummy vertices along edge $e$ into the same wall. Both algorithms take $O(|V|+|E|)$ time because each vertex is examined and assigned into a wall and this is done by examining the neighbours of the vertices, i.e. by examining all the edges of the digraph.

Lemma 1. Both the zig-zag wall partition and the dominating wall partition algorithms assign all dummy vertices along an edge to the same wall and partition the vertex set of the graph into two subsets in linear time.

\section{2 k-Wall Partitions}

We have designed a third algorithm for partitioning the vertex set into $k \geq 2$ walls according to $\mathrm{C} 4$. That is, the sum of spans of inter wall edges is kept small.

Similar to the two algorithms described above all the layers are scanned one by one from bottom to top. The first layer which contains more than one vertex is partitioned randomly and each following layer $L_{i}$ is partitioned on the basis of the partition of layer $L_{i-1}$.

For partitioning layer $L_{i}$ into $k$ subsets we apply Algorithm 4, In summary, for each vertex $u \in L_{i}$ all its immediate successors are considered, and $u$ is placed in the wall whose number is the closest integer to the average of the wall numbers of the immediate successors of $u$. In other words, the wall $u$ is placed in the barycenter of the walls its immediate successors are placed in. An example layout is shown in Figure 2(a).

In order to achieve a more even distribution of vertices between the walls, i.e. to satisfy $\mathrm{C} 1$, we can keep track on the number of vertices currently assigned to a wall and when we compute $b$ take this into account giving preference to the walls with fewer number of vertices. The implementation of such a procedure is presented in Algorithm 5 which is a generalised version of Algorithm 4 . Now the wall for vertex $u$ is computed as

$$
b=\left\lfloor\frac{\sum_{j=1}^{k} j * \max \left\{0, \text { neighbours }[j]-\left|L_{i}^{j}\right|\right\}}{\sum_{j=1}^{k} \max \left\{0, \text { neighbours }[j]-\left|L_{i}^{j}\right|\right\}}+0.5\right\rfloor .
$$





in the case $\sum_{j=1}^{k} \max \left\{0\right.$, neighbour $\left.s[j]-\left|L_{i}^{j}\right|\right\}>0$. Otherwise $u$ is placed in the wall with currently the fewest number of vertices in the current layer.

It is easy to see that Algorithm 4 guarantees that all dummy vertices along an edge belong to the same wall. However, this is no longer guaranteed with the proposed balancing technique. Thus, when applying the balancing technique we first need to check whether $u$ is dummy and if it is then to place it into the wall of its immediate successor. 
The time complexity of the proposed $k$-wall partitioning algorithm is also $O(|V|+|E|)$ because similar to the two-wall partitioning algorithms each vertex and each edge are scanned once.

Lemma 2. Both versions of the $k$-wall partition algorithm assign all dummy vertices along an edge to the same wall and partition the vertex set of the graph into $k \geq 2$ subsets in linear time.

\section{Computational Results}

We applied the presented layout technique for computing 3D hierarchical layouts of graphs taken from the Rome dataset 3 . We randomly chose ten graphs with 75 vertices. The number of edges of each of them is close to twice the number of vertices. In the remainder of this section we compare the different wall assignment techniques:

- (ZZ) Zig-zag wall partition.

- (DW) Dominating wall partition.

- (MC) Balanced min-cut wall partition introduced in 6].

- (KW) $k$-wall partition.

- (BW) Balanced $k$-wall partition.

- (SW) Single wall, i.e. a 2D hierarchical layout.

$\mathrm{MC}$ is a version of the balanced min-cut wall partition introduced in 6. Note that we have slightly modified the original algorithm to make sure all dummy vertices along an edge go to the same wall which we did not consider in our previous work.

We assumed that the input graphs are undirected and we assigned direction to each edge from the vertex with the higher degree to the vertex with the lower degree. The reason for doing this is because we had to assign the direction of the edges by using the same method in all experimental graphs. It also allows us to test how our 3D hierarchical drawings can be used for emphasising centrality in large and complex networks, in particular the degree centrality, by having a loose connection between the layers and the centrality values. If both endpoints of an edge have the same degree then the direction is assigned randomly. We applied Algorithm 1 six times, once for each wall assignment technique. Each time we used the same algorithms for the other four steps: cycle removal, layer assignment, vertex ordering, and coordinate assignment.

We remove directed cycles by reversing the direction of the back edges in a DFS tree of the digraph. For layer assignment we used the longest-path algorithm followed by an improvement heuristic [7. The vertex ordering step is performed with a layer-by-layer sweep and the barycenter heuristic taking into account the wall partition. Details about the vertex ordering with 2 walls can be found in 6]. We used a trivial extension of the same method for $k>2$ walls. For the coordinate assignment step we applied the Brandes-Köpf algorithm for each wall independently [1]. The $z$-coordinates are given by the wall numbers. 
Table 1. Vertex and edge distribution for two-wall partitions

\begin{tabular}{|c|c|c|c|c|c|c|c|c|c|}
\hline \multirow[t]{2}{*}{ graph } & \multicolumn{3}{|c|}{$\mathbf{Z Z}$} & \multicolumn{3}{|c|}{ DW } & \multicolumn{3}{|c|}{ MC } \\
\hline & vertices & edges & $\begin{array}{c}\text { inter } \\
\text { wall } \\
\text { edges }\end{array}$ & vertices & edges & $\begin{array}{c}\text { inter } \\
\text { wall } \\
\text { edges }\end{array}$ & vertices & edges & \begin{tabular}{|c|} 
inter \\
wall \\
edges
\end{tabular} \\
\hline 1 & 7730 & 7521 & 24 & 4265 & 4448 & 28 & 5552 & 5148 & 21 \\
\hline 2 & 5573 & 4775 & 32 & 4484 & 4370 & 41 & 6662 & 6364 & 27 \\
\hline 3 & 6346 & 5736 & 46 & 4168 & 4147 & 51 & 5455 & 5353 & 33 \\
\hline 4 & 6556 & 6151 & 33 & 4180 & 4067 & 38 & 6259 & 6257 & 26 \\
\hline 5 & 7958 & 7354 & 31 & 4988 & 5177 & 30 & 7067 & 7361 & 24 \\
\hline 6 & 8043 & \begin{tabular}{|l|}
8337 \\
\end{tabular} & 34 & 4380 & 4765 & 42 & 6558 & 6556 & 33 \\
\hline 7 & 10753 & 11946 & 30 & 6793 & 7780 & 38 & 8278 & 8579 & 31 \\
\hline 8 & 7351 & 6748 & 30 & 6064 & 6348 & 34 & 6262 & 5966 & 20 \\
\hline 9 & 9743 & 10438 & 26 & 6575 & 6955 & 44 & 7268 & $\begin{array}{|ll|}70 & 69 \\
\end{array}$ & 29 \\
\hline 10 & 7177 & 6578 & 43 & 5197 & 5781 & 48 & 7573 & 7977 & 30 \\
\hline
\end{tabular}

Table 2. Vertex and edge distribution for k-wall partitions

\begin{tabular}{|l||c|c|c|c|c|}
\hline \multirow{2}{*}{ graph } & \multirow{2}{*}{$\begin{array}{c}\text { number } \\
\text { of walls }\end{array}$} & \multicolumn{2}{|c|}{ KW } & \multicolumn{2}{c|}{ BW } \\
\cline { 3 - 6 } & vertices & edges & vertices & edges \\
\hline \hline $\mathbf{1}$ & 3 & 283940 & 223530 & 423431 & 262218 \\
\hline $\mathbf{2}$ & 3 & 464240 & 423539 & 464636 & 313525 \\
\hline $\mathbf{3}$ & 2 & 4861 & 3567 & 5851 & 5246 \\
\hline $\mathbf{4}$ & 3 & 384637 & 293927 & 434533 & 303320 \\
\hline $\mathbf{5}$ & 4 & 34393430 & 26332822 & 41293334 & 28182723 \\
\hline $\mathbf{6}$ & 4 & 33512019 & 26561312 & 42283122 & 30162410 \\
\hline $\mathbf{7}$ & 4 & 34534330 & 22523624 & 46424527 & 30303218 \\
\hline $\mathbf{8}$ & 3 & 404440 & 273831 & 394045 & 232636 \\
\hline $\mathbf{9}$ & 3 & 365846 & 265935 & 524244 & 403329 \\
\hline $\mathbf{1 0}$ & 3 & 466240 & 376735 & 545341 & 374430 \\
\hline
\end{tabular}

First we compare the two-wall partitioning algorithms. Table 1 shows the vertex and edge distribution between the walls as well as the number of inter wall edges. It can be observed that MC does really find wall partitions which have more even distribution of vertices and edges between the two walls. The MC partitions typically have the minimum number of inter wall edges, which is what we expected. The advantage of $\mathrm{ZZ}$ and DW is the special arrangement of inter wall edges they guarantee. We can also see that the distribution of vertices and edges between the two walls in ZZ and DW is not necessarily unbalanced.

Table 2 compares the vertex and edge distribution for $\mathrm{KW}$ and BW. The number of walls $k$ is a half of the number of layers $h$, i.e. $k=\left\lfloor\frac{h}{2}\right\rfloor$. Thus, different graphs have a different number of walls. We can observe that BW does find more balanced distribution of vertices between the walls only if the $\mathrm{KW}$ distribution is not balanced (e.g., graph 9). When the KW distribution is relatively well balanced then BW may perform worse than KW (e.g., graph 8). 
Table 3. Total span of inter wall edges in k-wall partitions

\begin{tabular}{|l||c|c|c|c|c|}
\hline \multirow{2}{*}{ graph } & number & \multicolumn{2}{|c|}{ KW } & \multicolumn{2}{c|}{ BW } \\
\cline { 3 - 6 } & & $\begin{array}{c}\text { of walls } \\
\text { wall edges }\end{array}$ & $\begin{array}{c}\text { span of inter } \\
\text { wall edges }\end{array}$ & $\begin{array}{c}\text { number of inter } \\
\text { wall edges }\end{array}$ & $\begin{array}{c}\text { span of inter } \\
\text { wall edges }\end{array}$ \\
\hline \hline $\mathbf{1}$ & 3 & 33 & 33 & 54 & 68 \\
\hline $\mathbf{2}$ & 3 & 47 & 47 & 63 & 78 \\
\hline $\mathbf{3}$ & 2 & 37 & 37 & 41 & 41 \\
\hline $\mathbf{4}$ & 3 & 50 & 50 & 62 & 77 \\
\hline $\mathbf{5}$ & 4 & 49 & 57 & 62 & 94 \\
\hline $\mathbf{6}$ & 4 & 47 & 57 & 74 & 105 \\
\hline $\mathbf{7}$ & 4 & 61 & 69 & 85 & 118 \\
\hline $\mathbf{8}$ & 3 & 49 & 49 & 60 & 73 \\
\hline $\mathbf{9}$ & 3 & 48 & 48 & 66 & 80 \\
\hline $\mathbf{1 0}$ & 3 & 47 & 47 & 75 & 93 \\
\hline
\end{tabular}

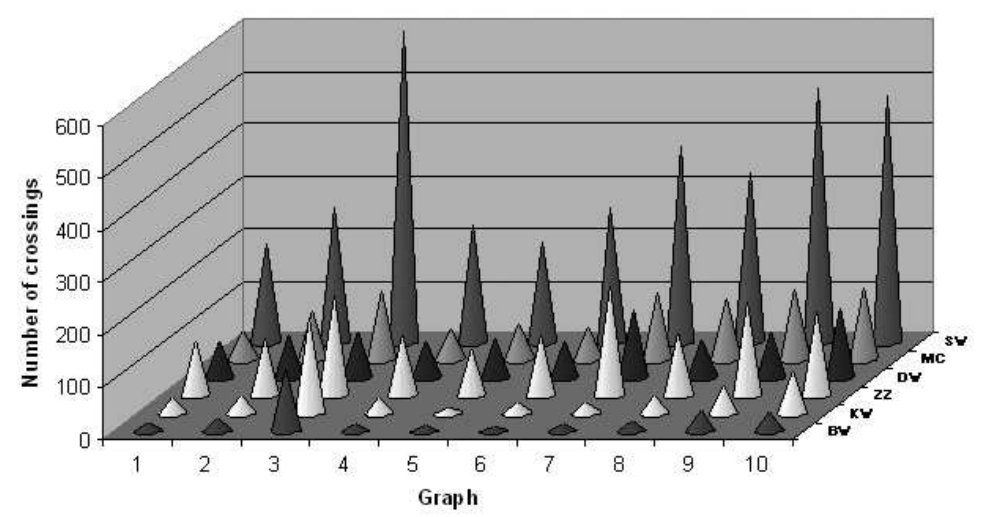

Fig. 3. Total number of edge crossings between intra wall edges (in all walls)

Table 3 compares KW and BW in terms of sum of the span of inter wall edges. KW performs better than BW. We observed that the $\mathrm{KW}$ wall partitions have very few inter wall edges between non-adjacent walls.

We have also computed the total number of crossings between intra wall edges. The results are presented in Figure 4. As expected, the bigger the number of walls the fewer crossings between intra wall edges. The best of the two-wall partitions is DW which typically results in a fewer number of intra wall edge crossings than ZZ and MC. The fewest number of intra wall edge crossings is reached in BW which is a result we did not expect. However, the BW partitions have a larger number of inter wall edges and a larger sum of spans of inter wall edges than KW (see Table 3 ).

\section{Conclusions and Current Work}

We introduced a 3D extension to the Sugiyama method. We propose to partition the vertex set into $k \geq 2$ walls; each wall contains a $2 \mathrm{D}$ drawing of a layered 
digraph. This is done by introducing an additional wall assignment step into the Sugiyama method after the layer assignment step. We propose three different wall assignment algorithms which meet different optimisation criteria.

The computational results suggest that the proposed 3D graph drawing convention results in reduced visual complexity. The choice of a particular wall assignment algorithm may highly depend on the interaction and navigation techniques. It is also possible to develop new vertex ordering heuristics specific for the 3D layered graph drawings with $k$ walls. We are also looking at the possibility of defining new optimisation problems arising from 3D drawing aesthetic criteria.

Acknowledgement. The authors would like to thank Michael Forster for the valuable discussion and suggestions as well as for implementing the Brandes-Köpf algorithm for coordinate assignment which we used in the drawings included in the paper.

\section{References}

1. U. Brandes and B. Köpf. Fast and simple horizontal coordinate assignment. In P. Mutzel, M. Jünger, and S. Leipert, editors, Graph Drawing: Proceedings of 9th International Symposium, GD 2001, volume 2265 of Lecture Notes in Computer Science, pages 31-44. Springer-Verlag, 2002.

2. G. Di Battista, P. Eades, R. Tamassia, and I. G. Tollis. Graph Drawing. Prentice Hall, 1999.

3. G. Di Battista, A. Garg, G. Liotta, R. Tamassia, E. Tassinari, and F. Vargiu. An experimental comparison of four graph drawing algorithms. Computational Geometry: Theory and Applications, 7:303-316, 1997.

4. P. Eades and K. Sugiyama. How to draw a directed graph. Journal of Information Processing, 13(4):424-437, 1990.

5. A. Garg and R. Tamassia. GIOTTO: A system for visualizing hierarchical structures in 3D. In S. North, editor, Graph Drawing: Symposium on Graph Drawing, GD '96, volume 1190 of Lecture Notes in Computer Science, pages 193-200. Springer-Verlag, 1997.

6. S.-H. Hong and N. S. Nikolov. Layered drawings of directed graphs in three dimensions. In S.-H. Hong, editor, Information Visualisation 2005: Asia-Pacific Symposium on Information Visualisation (APVIS2005), volume 45, pages 69-74. CRPIT, 2005.

7. N. S. Nikolov and A. Tarassov. Graph layering by promotion of nodes. Special issue of Discrete Applied Mathematics associated with the IV ALIO/EURO Workshop on Applied Combinatorial Optimization, to appear.

8. D. Ostry. Some three-dimensional graph drawing algorithms. Master's thesis, University of Newcastle, 1996.

9. K. Sugiyama, S. Tagawa, and M. Toda. Methods for visual understanding of hierarchical system structures. IEEE Transaction on Systems, Man, and Cybernetics, 11(2):109-125, February 1981.

10. C. Ware and G. Franck. Viewing a graph in a virtual reality display is three times as good as a 2D diagram. In IEEE Conference on Visual Languages, pages 182-183, 1994. 